\section{Dampfeinsparung durch periodische Katalysator-Reaktivierung bei der Styrol-Synthese}

\section{Junguk Choi und Gerhart Eigenberger*}

Herrn Professor Dr. Dr. h. c. Ewald Wicke zum 75. Geburtstag

Der größte Teil des weltweit in ca. 40 Mio. t/a produzierten Styrols entsteht durch Dehydrieren von Ethylbenzol. Dabei wird dampfförmiges Ethylbenzol in Gegenwart von Wasserdampf bei Temperaturen um $600^{\circ} \mathrm{C}$ an $\mathrm{KOH}$-dotierten Eisenoxid-Katalysatoren gemäB Gl. (1) zu Styrol und Wasserstoff gespalten [1]:

$\mathrm{C}_{8} \mathrm{H}_{10} \rightleftharpoons \mathrm{C}_{8} \mathrm{H}_{8}+\mathrm{H}_{2} \quad, \quad \Delta h_{\mathrm{R}}=+124,9 \mathrm{~kJ} / \mathrm{mol}$

Neben der Hauptreaktion spielen unter technischen Bedingungen die Desalkylierung von Ethylbenzol zu Benzol, die Toluol-Bildung und die Bildung von Styrol-Oligomeren eine Rolle. Die Styrol-Oligomere können den Katalysator verkoken und zu einer schnellen Desaktivierung führen, wenn sie nicht vom Katalysator entfernt werden. Deswegen wird dem Reaktionsgemisch Wasserdampf zugesetzt, der höhermolekulare Ablagerungen in einer Vergasungsreaktion gemäß Gl. (2) umwandelt:

* Dipl.-Ing. J. Choi und Prof. Dr.-Ing. G. Eigenberger, Institut für Chemische Verfahrenstechnik der Univ. Stuttgart, Böblinger Str. 72,7000 Stuttgart 1.
$\left(\mathrm{C}_{8} \mathrm{H}_{8}\right)_{\mathrm{n}}+8 n \mathrm{H}_{2} \mathrm{O} \rightarrow 8 n \mathrm{CO}+12 n \mathrm{H}_{2}$,

$\mathrm{CO}+\mathrm{H}_{2} \mathrm{O} \rightleftharpoons \mathrm{CO}_{2}+\mathrm{H}_{2}$.

Typische Industrie-Katalysatoren verlangen für verkokungsfreien Betrieb ein Gewichtsverhältnis Wasserdampf zu Ehtylbenzol von $1: 1$ bis $1: 2,5$.

Reaktionstechnisch hat die Wasserdampfzugabe den weiteren Vorteil, daß sie den Styrol-Partialdruck senkt und damit den Gleichgewichtsumsatz erhöht. Gravierender ist aber der Nachteil, daß mit steigendem Wasserdampfanteil der Energieaufwand für die Aufheizung des Reaktorzulaufs und die Kondensation des Ablaufs deutlich zunimmt. Daher wird die Styrol-Synthese bei dem niedrigsten Wasserdampfverhältnis gefahren, das einen desaktivierungsfreien Betrieb erlaubt. Gegebenenfalls kann der Katalysator durch vorübergehenden Betrieb mit reinem Wasserdampf wieder reaktiviert werden [2].

Im folgenden wird über eine Serie von Versuchen berichtet, durch periodische Änderung des Wasser/Ethylbenzol-Verhältnisses zu einem im Mittel niedrigeren Wasserdampfanteil als bei stationärer Betriebsweise zu gelangen.

\section{Versuchsanlage}

Die Versuche wurden in einer Laboranlage nach Abb. 1 durchgeführt. Als Reaktor dient ein salzschmelze-beheiztes Reaktionsrohr von $20 \mathrm{~mm}$ Innendurchmesser. Die Höhe der Katalysator-Schüttung beträgt $2 \mathrm{~m}$ (Katalysator-Menge $627 \mathrm{~g} \bumpeq 615 \mathrm{ml}$ ); darüber befinden sich $0,5 \mathrm{~m}$ inerte Vorschüttung zum Aufheizen des Zulaufs. Der Katalysator der Fa. BASF AG besteht aus Vollsträngen von ca. $2 \mathrm{~mm} \times$ 


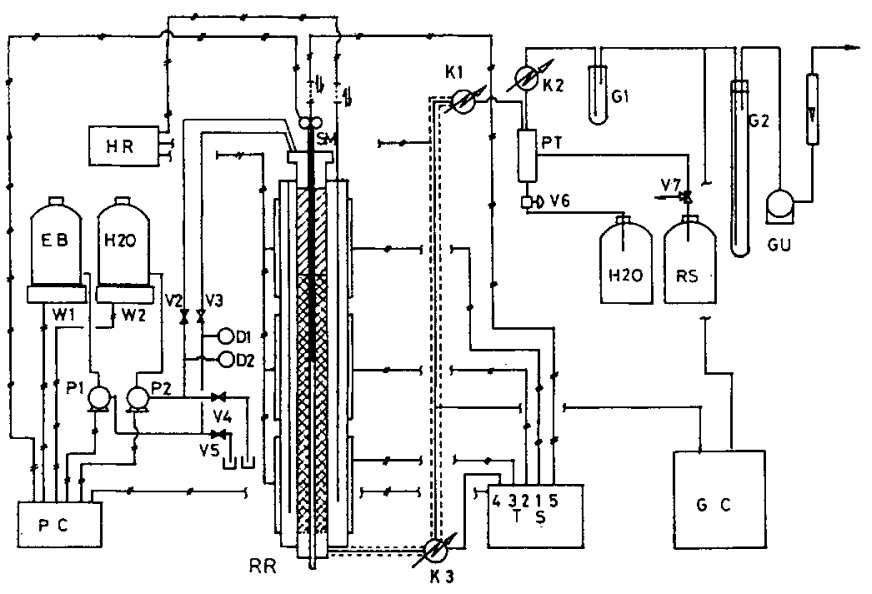

Abb. 1. Schema der Versuchsanlage.

$\begin{array}{llll}D 1, D 2 & \text { Druckmesser, } & P C & \begin{array}{l}\text { Personal Computer, } \\ \text { Phasentrenner, }\end{array} \\ G C & \text { Ethylbenzol, } & P T & \begin{array}{l}\text { Rohrreaktor mit } \\ \text { Gaschromatograph, }\end{array} \\ G 1, G 2 \text { Tauchrohre, } & & \begin{array}{l}\text { Salzbadheizung, } \\ \text { Rohstyrol, }\end{array} \\ G U & \text { Gasuhr, } & R S & \text { Schrittmotor, } \\ H R & \text { Heizungsregler, } & S M & \text { Temp.-Schreiber, } \\ K 1 & \text { Wasserkühler, } & T S & V 1-V 7 \text { Ventile, } \\ K 2 & \text { Tieftemp.-Kühler, } & W 1, W 2 \text { Waagen. } \\ K 3 & \text { Luftkühler, } & \end{array}$

$4 \mathrm{~mm}$. Reines Ethylbenzol und Wasser werden über getrennt ansteuerbare Dosierpumpen dem Reaktor zugeführt und verdampfen im Eingangsbereich des Reaktionsrohres. Die Dosierung wird gewichtskontrolliert über einen PC gesteuert. Bei allen nachstehend beschriebenen Versuchen betrug der Gesamtdurchsatz von Wasser und Ethylbenzol $700 \mathrm{~g} / \mathrm{h}$. Das entspricht bei einem Wasser/EthylbenzolVerhältnis im Zulauf von $1: 1$ einem LHSV-Wert ("liquid hourely space velocity $\left.{ }^{\prime}\right)$ von 0,661 Ethylbenzol/(l Katalysator $\cdot h$ ). Das jeweilige Wasserverhältnis ist als $W / E B$-Wert bei den Versuchsergebnissen vermerkt. Der Reaktorauslauf kann entweder direkt gasförmig oder nach Kondensation und Auftrennung in gasförmige, wäßrige und organische Phase gaschromatographisch analysiert werden.

Abb. 2 zeigt ein typisches Profil der Reaktortemperatur, gemessen mit einem zentral im Reaktor angeordneten, verschiebbaren Thermoelement. Der Beginn der aktiven Schüttung ist deutlich am Temperaturabfall der endothermen Reaktion erkennbar. Der Temperaturverlauf im salzschmelze-beheizten Reaktormantel ist isotherm.

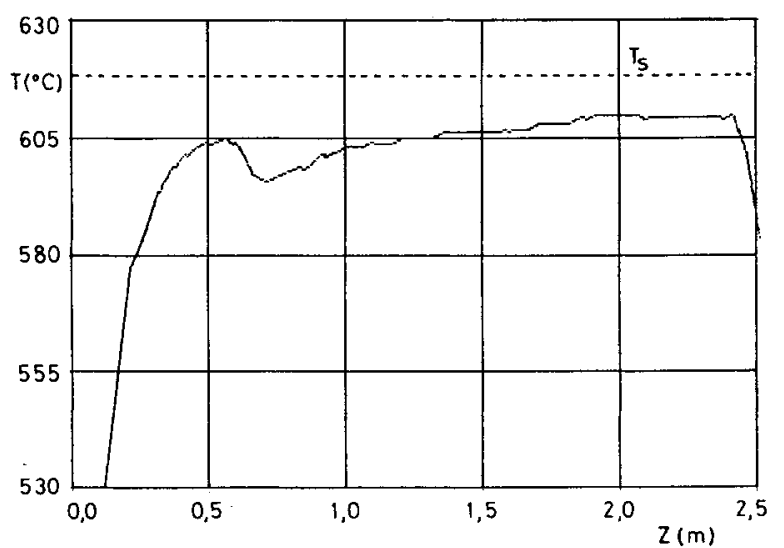

Abb. 2. Typischer Temperaturverlauf in Rohrmitte und im Heizmantel $\left(T_{\mathrm{S}}=615^{\circ} \mathrm{C}, W / E B=0,8, X_{\mathrm{EB}}=43,7 \%, S_{\mathrm{St}}=96,1 \%\right)$.

\section{Versuchsergebnisse}

Ausgangspunkt des Versuchsprogramms war die Beobachtung, daB ein vollständig mit Wasserdampf regenerierter Katalysator über einen längeren Zeitraum hinweg hohe Umsätze mit niedrigem Wasserdampfverhältnis zuläßt, bevor eine Desaktivierung eintritt. Ein typisches Beispiel zeigt Abb. 3. Aufgetragen sind die gemessenen Ethylbenzol- und Styrol-Konzentrationen in der organischen Phase des Reaktoraustrags über der Zeit. Die Mittelwerte für EthylbenzolUmsatz $X_{\mathrm{EB}}$ und Styrol-Selektivität $S_{\mathrm{St}}$ sind ebenfalls vermerkt. Man erkennt, daß sowohl bei 0,5 als auch bei 0,7 Teilen Wasserdampf eine mehrstündige Betriebsperiode mit hohem Ethylbenzol-Umsatz möglich ist, bevor die Desaktivierung einsetzt. Das steht im Gegensatz zum Verhalten eines "eingefahrenen“ Katalysators, der sofort auf Änderungen im Wasserdampfverhältnis reagiert. Abb. 4 zeigt entsprechende Zeit/Umsatz-Verläufe.
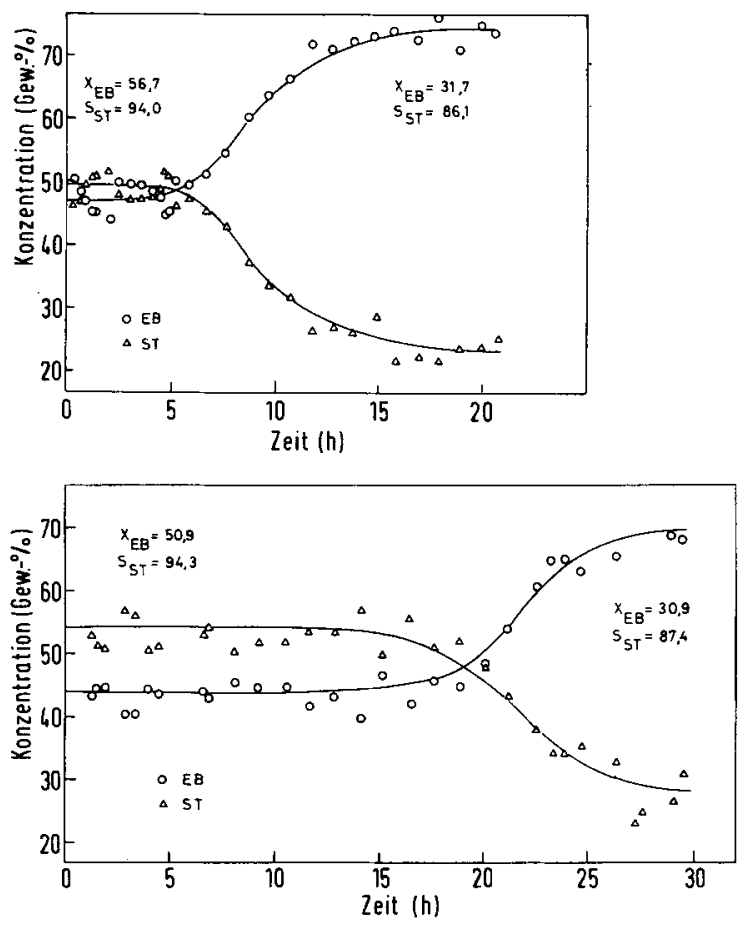

Abb. 3. Konzentrations/Zeit-Verlauf im organischen Produkt bei vollständig wasserdampf-regeneriertem Katalysator. a) (oben) nach Regenerierung mit $240 \mathrm{~g}$ Wasser/h über $2,3 \mathrm{~h}$ und Betrieb mit $W / E B$ $=0,5, T_{\mathrm{S}}=615^{\circ} \mathrm{C} ; \mathrm{b}$ ) (unten) nach Regenerierung mit $200 \mathrm{~g}$ Wasser $/ \mathrm{h}$ über $0,5 \mathrm{~h}$ und Betrieb mit $W / E B=0,7, T_{\mathrm{S}}=615^{\circ} \mathrm{C}$.

In einer Reihe von Versuchen wurde dieser Sachverhalt bestätigt. Demnach reagiert auch ein mit hohem WasserdampfüberschuB (z. B. $2: 1)$ betriebener Katalysator sofort auf eine Absenkung des Wasserverhältnisses auf $z$. B. 0,7, während bei einem vollständig regenerierten „frischen “ Katalysator eine Induktionsperiode von vielen Stunden zu beobachten ist.

In einer folgenden Versuchsserie wurde getestet, welche Regenerierbedingungen für eine "vollständige ${ }^{\mu}$ Regenerierung mindestens einzuhalten sind. Bei willkürlich festgelegter Regenerierzeit von $0,5 \mathrm{~h}$ wurde die Regenerierwassermenge variiert.

Das Ergebnis ist in Abb. 5 als Anfangsumsatz über der Regenerierwassermenge dargestellt. Demnach reichen unter den Versuchsbedingungen $100 \mathrm{~g}$ Wasser für eine vollständige Regenerierung aus. In weiteren Versuchsserien wurde untersucht, ob durch kurzperiodische Änderungen des Wasser/Ethylbenzol-Verhältnisses im Sinne einer periodischen Reaktionsführung [3] ein Dauerbetrieb mit im Mittel reduziertem Wasseranteil möglich ist. Ein typisches Versuchsergebnis zeigt Abb. 6. Auffallend sind die stark schwankenden Konzentrationsverläufe und die Tatsache, daß unter den gewählten Bedingungen nach einer Induktionszeit von ca. $45 \mathrm{~h}$ ebenfalls ein Ubergang in den desaktivierten Zustand auftritt.

Eine Erklärung für die stark schwankenden Konzentrationswerte liefert Abb. 7, in der für eine ähnliche periodische Betriebsweise der ge- 


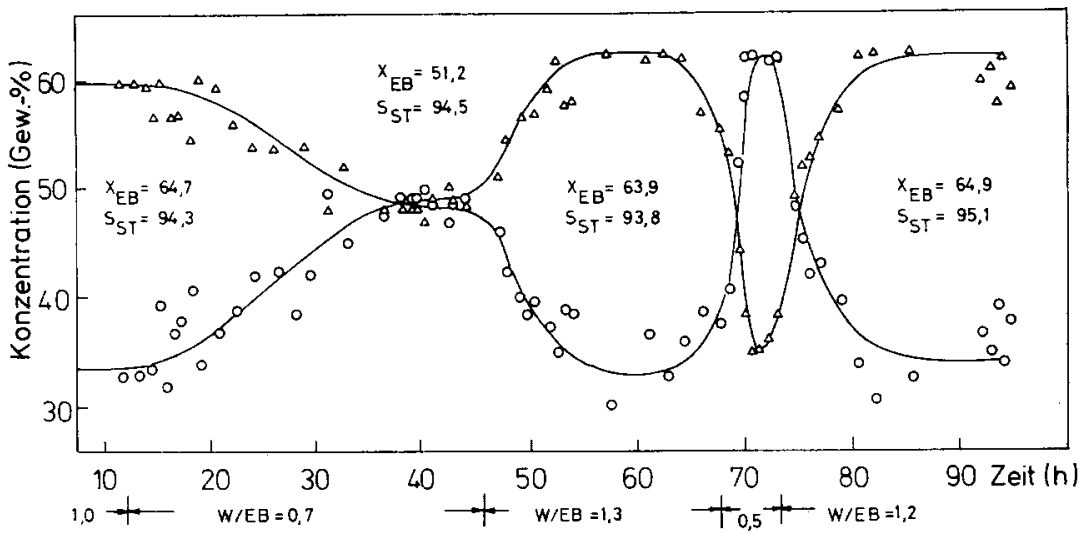

Abb. 4. Konzentrations/Zeit-Verlauf im organischen Produkt bei eingefahrenem Katalysator und Änderungen im Wasser/Ethylbenzol-Verhältnis $(W / E B) ; T_{\mathrm{S}}=625^{\circ} \mathrm{C}$.

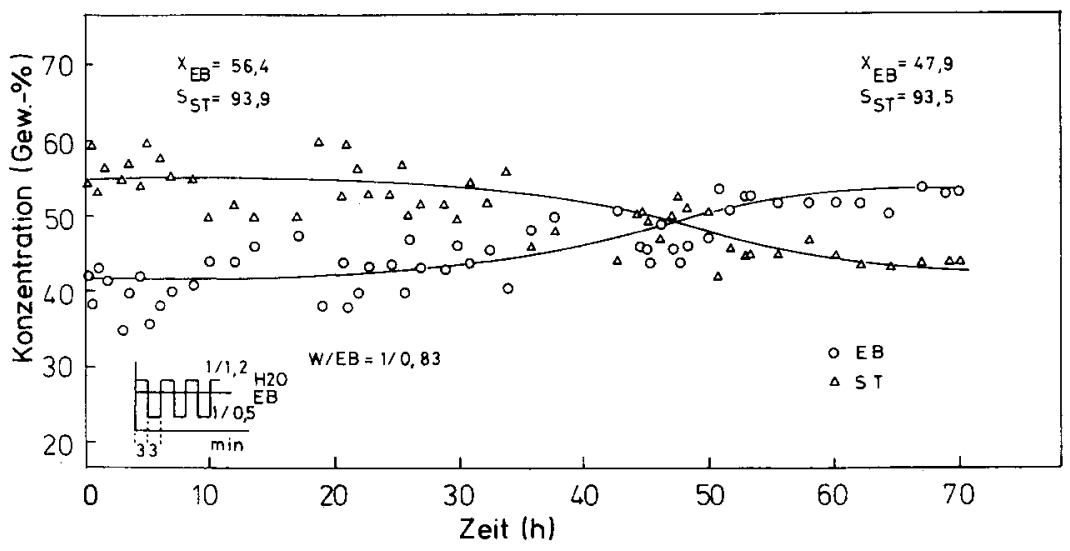

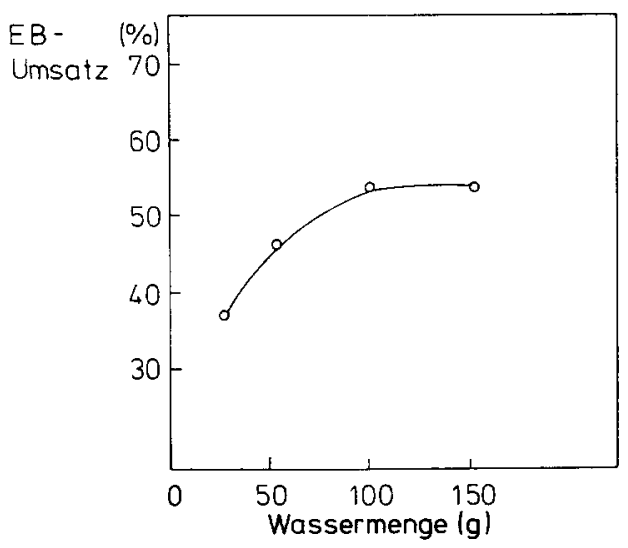

Abb. 5. Anfangsumsatz über Regenerierwassermenge bei $1 / 2$-stündiger Regenerierung und nachfolgendem Betrieb mit 0,7 Teilen Wasserdampf; $T_{\mathrm{S}}=615^{\circ} \mathrm{C}$. messene Konzentrationsverlauf in der organischen Phase des Reaktoraustrags sowie im Abgas während einer Periode aufgetragen ist. Man erkennt die ausgeprägte periodische Änderung der Konzentrationen, die mit den Analyse-Zeitpunkten in Abb. 6 nicht synchronisiert war.

Der Verlauf von Ethylbenzol- und Styrol-Konzentration in Abb. 7 könnte auf einen im Mittel hohen Ethylbenzol-Umsatz schließen lassen. Tatsächlich wird während dem Teil der Betriebsperiode mit ethylbenzol-freiem Zulauf nur sehr wenig Styrol, allerdings mit hoher Konzentration, ausgetragen. Daher liegen die erzielten mittleren Umsätze und Selektivitäten im Bereich der im stationären Betrieb erhaltenen Werte.

\section{Zusammenfassung und Interpretation}

Die hier vorgestellten Versuchsergebnisse können wie folgt zusammengefaßt werden: Verglichen mit einem eingefahrenen, stationär betriebenen Katalysator erlaubt ein vollständig wasserdampfregenerierter Katalysator über eine begrenzte Zeit („Induktionsperiode ${ }^{\omega}$ ) hinweg einen Betrieb mit einem deutlich reduzierten Wasserdampfanteil. Da die für die vollständige Regenerierung erforderliche Wasserdampfmenge bei den untersuchten Bedingungen mit $160 \mathrm{~g} / \mathrm{kg}$ Kat. niedrig ist, läßt sich durch periodische, vollständige Regenerierung ein im Mittel deutlich niedrigerer Wasserdampfverbrauch als bei stationärem Betrieb erreichen. Verglichen mit einem kurzperiodischen Betrieb mit einer Periodendauer im Minutenbereich erscheint ein längerperiodischer Betrieb mit einer Periodendauer zwischen 5 und $20 \mathrm{~h}$ sowohl reaktions- als auch betriebstechnisch günstiger.

Einem Vorschlag von $E$. Wicke folgend [4] läßt sich die Tatsache der induktionsgehemmten Desaktivierung des Styrol-Katalysators dadurch erklären, daß sich höhermolekulare Reaktionsprodukte vor allem an solchen Stellen der Katalysator-Oberfläche anlagern, an denen sich bereits gleichartige Keime befinden. Wie in anderen Berei-
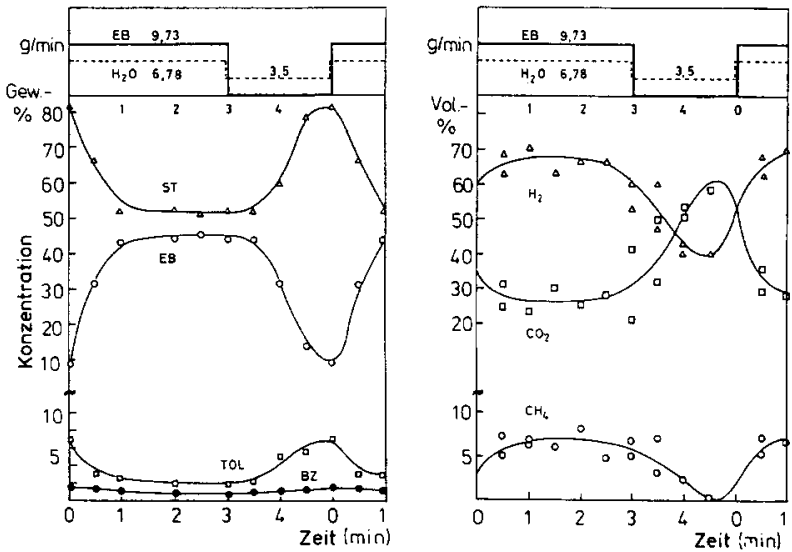

Abb. 7. Konzentrations/Zeit-Verläufe bei der gezeigten periodischen Betriebsweise mit intervallweiser Ethylbenzol-Zufuhr und sprungförmiger Änderung der Wasserdampfmenge; links: organisches Produkt, rechts: Dehydriergas.

chen der Physikalischen Chemie ist aber die Keimbildung selbst ein Vorgang, der mit einer sehr viel kleineren Geschwindigkeit abläuft als das Wachstum. Die Umsetzung dieser Vorstellung in ein kinetisches Modell mit einem autokatalytischen Ansatz für die KatalysatorDesaktivierung zeigt, daB damit das Desaktivierungsverhalten zutreffend wiedergegeben werden kann [5].

Der BASF AG, Ludwigshafen/Rh., sei für die Bereitstellung von Katalysator und Ethylbenzol für die durchgeführten Untersuchungen gedankt.

Eingegangen am 6. März 1989 [K 1041] 


\section{Literatur}

[1] Lee, H. E.: Catal. Rev. Sci. Eng. 8 (1973) S. 285/305.

[2] Herzog, B. D.; Rase, H. F.: Ind. Eng. Chem., Prod. Res. Dev. 23 (1984) S. 187/196.
[3] Renken, A.: Chem.-Ing.-Tech. 54 (1982) S. 571/580.

[4] Wicke, E.: Persönliche Mitteilung.

[5] Jungnitz, $M$. .: Diplomarbeit am Institut für Chemische Verfahrenstechnik der Univ. Stuttgart, 1988. 